\title{
Research on Mathematical Model for Idle Speed Control of Engine
}

\author{
Sun Jianmin ${ }^{1, a}$, Yin Fengfeng ${ }^{2, b}$ and Wei Zhiyuan ${ }^{3, c}$ \\ ${ }^{1}$ School of Mechanical-electronic and Automobile Engineering, Beijing University of Civil \\ Engineering and Architecture, China \\ ${ }^{2}$ School of Mechanical-electronic and Automobile Engineering, Beijing University of Civil \\ Engineering and Architecture, China \\ ${ }^{3}$ School of Mechanical-electronic and Automobile Engineering, Beijing University of Civil \\ Engineering and Architecture,China \\ ajinmin sun@tum.com, bm17888812565@163.com, c15901127824@163.com
}

Keywords: engine control, idle condition, modeling method

\begin{abstract}
The modeling method is commonly used in the simulation of engine at home and abroad, the engine which is used for auto control and performance analysis model. It includes static and dynamic modeling methods, such as engine nonlinear autoregressive model, the average model, the chart model and hybrid model. .In the actual process of modeling, we should make a trade-off between simplicity and accuracy of the model, select the appropriate modeling method for the specific problem.
\end{abstract}

\section{Introduction}

Idle condition is one of the main working condition of the engine.From the Olympic Games in Beijing in 2008, advocating the green Olympic Games and the blue Olympic, to the APEC meeting in November 2014, Beijing, tianjin and hebei province implement the APEC blue.According to statistics.The idle speed will increase fuel consumption, causing unnecessary waste when it is is too high,While the idle speed is too low to make obvious reinforcement effect of exhaust gas dilution of mixture in cylinder, if a slight change of load or resistance will cause instability or even shut down engine operation.Therefore, in order to meet the requirements of increasingly stringent emission regulations, as far as possible,we should reduce the idle speed and maintain the stability of idle speed appears especially important.But the engine idle speed control system is the most important in the electronic control system and it is also one of the most complicated part, researchers have a lot of research on the idle speed control around the world, however, its performance is still not satisfactory.

Modern cars are generally equipped with idle speed control system, controlled by the ECU and maintained the engine idle speed at a steady speed range.Through the idle speed control valve to control the air inflow of engine, in order to make the engine to maintain the best idle speed running. The purpose of the idle speed control is to control the engine speed near the best speed.

\section{Idle Speed Control System Modeling Method}

The key of the designe of Gasoline engine controller and the research of engine idle speed control strategy is to establish a relatively effective engine idle speed model, domestic idle model usually include NARX (nonlinear autoregressive) model, the chart model, foreign idle model usually include hybrid model, sliding model, linear model and average model and so on.Because the engine has a strong nonlinearity, hysteresis and slow time-varying characteristics, it is difficult to obtain accurate mathematical model of engine idle speed. However, because the change of the dynamic variables has a smaller engine in idle condition, so may be duly simplified engine model to adapt to the needs of the control algorithm.As shown in Fig. 2 of the idle speed control of the dynamic model is a simplified model of the engine idle speed control. 


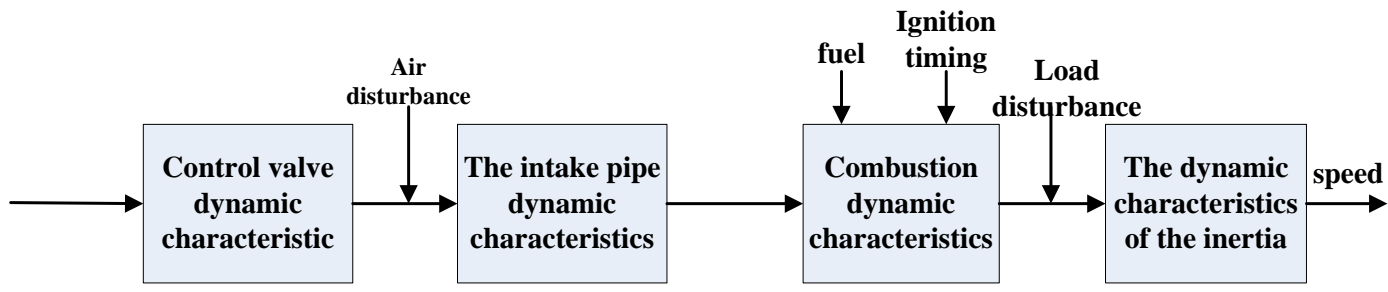

Fig. 2 A Dynamic Model of The Idle Speed Control

\subsection{Nonlinear Autoregressive (NARX) Model}

As the car engine idle process as A black box, its input for the solenoid valve opening (percentage), ignition advance Angle (Angle relative to the top dead center), air-fuel ratio (A/F, EGR flow, all kinds of known disturbance (e.g., air conditioning, electric Windows and doors, the opening of the electric equipment such as automatic transmission), and other unknown disturbances (such as fuel oil wet wall effect, the vibration caused by input parameter perturbation, and element aging caused by the structure parameter perturbation, etc.).The output for the idle speed is N.Consider only the electromagnetic valve opening $\phi$ and ignition advance angle $\theta$ two input variables and output of the crankshaft rotational speed $\mathrm{n}$, the idle process NARX model are as follows:

$$
\mathrm{n}(k)=\sum_{i=1}^{r} a_{i} X_{i}(k)
$$

In [2], a class of nonlinear autoregressive model is adopted to describe oil supply, ignition advance angle and the relationship between the crankshaft speed in the idle speed the process, but also the use of the identification algorithm, a modified 125 engine idle speed test data processing, the measured speed was consistent with the model prediction output.in[3],it is introduced step by step optimization method to calculate the approximate optimal model.Using a class of nonlinear autoregressive model, describes the idle idle speed in the process of oil, ignition advance Angle and the relationship between the crankshaft speed.In [4], according to the physical model of engine and static pulse spectrum diagram, for a 4 cylinder, 2 litres of spark-ignited gasoline engine built a nonlinear model of the average engine idle speed.Characteristics of the model is in the idle speed set-point linearization, and considered the parameter uncertainties, the engine torque output lagging and external disturbance. Combined with the established model, the delay dependent robust $\mathrm{H} \infty$ state feedback controller is put forward to guarantee the asymptotic stability of the system, and make the system has a certain robust $\mathrm{H} \infty$ performance. In the en-DYNA engine model of simulation prove the validity of the modeling and control methods.

\subsection{The Graph Model}

Graph model's the main characteristics is that model calculation is given priority to with look-up table and the fitting formula, the establishment of the model mainly depends on the fitting of experimental data, this model has the advantage of simple structure, small amount of calculation and simulation process only needs to be look-up table and interpolation, the engine working condition of the steady state characteristics simulation can achieve higher precision of faults are resolved, it is not conducive to the design of the control system, and the established model generally applies only to a specific engine because all kinds of chartsand the establishment of the fitting formula is based on the specific engine test in the process of building model of, this type of engine model can be thought of a model based on test.The model characteristic has carried on the comprehensive description of the engine, the fuel supply carburetor or fuel injection, air inlet pipe, energy conversion and power output of four parts. The input of the model is the throttle opening, air fuel ratio, ignition advance angle and load torque output of the engine speed, models of each sub models exist in the form of chart, data is derived from a lot of test.This model actually emphasis on the steady state characteristics of engine simulation, description of the engine dynamic characteristics only considering the time delay and the 
working process of the intake manifold absolute pressure and engine speed increment of integral.Engine chart model is shown in Fig. 3.

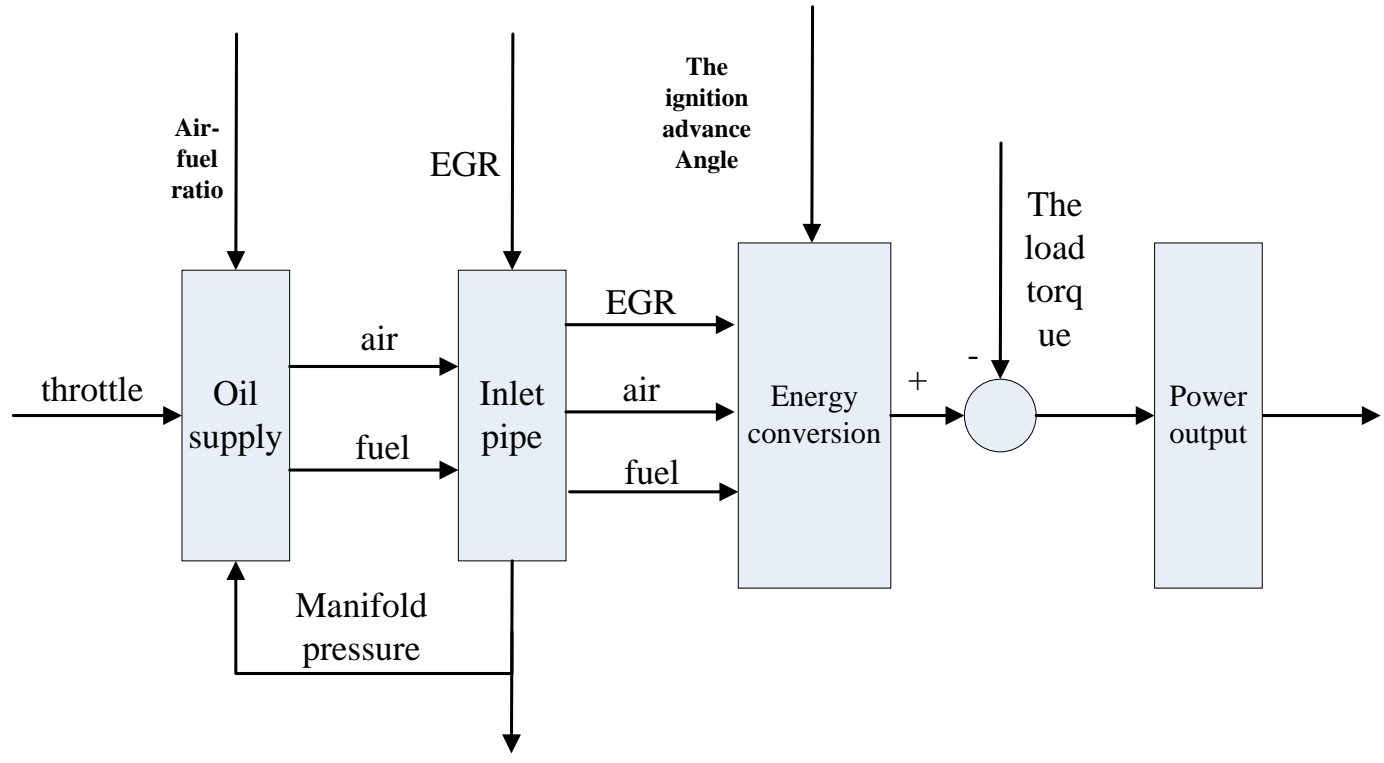

Fig. 3 Engine Graph Model

\subsection{The Average Model}

Aquino in 1981 [5] put forward the average model, the model of engine characteristic has carried on the comprehensive description, the advantage of this model is relatively simple modeling process, but it is not suitable for the engine state change of each stroke cases.

In 1989, Hendricks put forward an average three states dynamics model, the model for the three dynamic sub models: intake manifold air flow submodel, fuel steam and oil film model and power output module, the state space model can be described by the Eq. $1 \sim$ Eq. 3:

$$
\begin{aligned}
& \ddot{m_{f f}}=\frac{1}{\tau_{f}}\left(-\dot{m}_{f f}+X \dot{m}_{f t}\right) \\
& \dot{n}=\frac{H_{u}}{n I} \eta_{i}(n, p, \theta) \dot{m}_{f}\left(t-\tau_{d}\right)-\frac{P_{f}(n)+P_{p}(n, p)+P_{b}(n)}{n I} \\
& \dot{p}=\frac{R T}{V}\left(\dot{m}_{a t}(\alpha)-\dot{m}_{a p}\right)=-\frac{p}{\tau_{m}(n, p)}+\frac{R T}{V} \dot{m}_{a t}(\alpha)
\end{aligned}
$$

There are three main input: the throttle opening $\alpha$, flow fuel injection $m_{f t}$ and ignition advance angle $\theta$, three state variables of oil film mass flow rate $\ddot{m}_{f f}$, rotating speed of the crankshaft $\mathrm{n}$ and intake manifold pressure $m_{f t}$.

In [6],it discusse the engine model control and put forward based on the circulation of average discrete modeling method from the angle domain.It compared with now real-time simulation of the model and analyzes the different features of these models and the model in this paper.The example illustrates set up based on the circulation in Angle domain average model,it can better reflect the discrete nature of the engine.

\subsection{Hybrid Model}

In 2000, the Italian Andrea Balluchi put forward for the first time the engine idle speed condition of hybrid model [7, 8], they pointed out in the article, in order to meet the passengers of vehicle comfort, safety, fuel economy and emissions requirements increasing.The engine control system 
should be considered more robust technology and precise cycle model and this engine model should be considerd both the continuous time dynamic, and contains a mixture of discrete event model.

Gasoline engine has three main subsystems of intake, cylinder, crank shaft subsystem.Its mathematical description are:

Air intake subsystem: $S(\alpha)=a_{s} \alpha^{2}+b_{s} \alpha+c_{s}$

Cylinder subsystem: $\dot{p}(t)=a_{p} f_{\text {out }}(p, n)+b_{p} S(\alpha), f_{\text {out }}(p, n)=\left(G_{n p} n+O_{n p}\right) p+G_{n n} n+O_{n n}$

The crankshaft subsystem: $\dot{n(t)}=a_{n}^{c} n+b_{n}^{c}\left(T_{g}-T_{l}\right)$

The hybrid model consists of a series of discrete modules, continuous variables and the symbolic constant.System control input is the throttle opening $\alpha$, ignition advance angle $\theta$, disturbance inputs as the load torque ${ }^{T_{l}}$ and the clutch position ${ }^{b_{n}}$.So there are two types of input in the system: one is the continuous variable input $\alpha$ and $T_{l}$, the other one is the dynamics of continuous variable $\beta$ and $b_{n}$, the two decided to discrete model transformation, continuous variable reset and symbolic constant Settings. The mixed model of gasoline engine is a diverse group of:

$H=\left(\{Q, X, \Xi\},\left\{\sum_{C}, U\right\},\left\{M_{C}^{\text {disc }}, M_{C}^{c t s}\right\},\left\{\sum_{C}, D\right\},\left\{M_{e}^{\text {disc }}, M_{e}^{c t s}\right\},\{f, \delta\}\right)$

Among them:

Finite state model set: $Q=\left\{S_{-}, S, S_{+}, S_{-}^{L}, S^{L}, S_{+}^{L}\right\}$

Continuous time dynamics variables: $X=\left\{(P, N, \theta) \mid(P, N, \theta) \in I R^{3}\right\}$

Finite state agencies variables: $\Xi=\left\{\left(T, m_{c}, m_{e}, \varphi\right) \mid\left(T, m_{c}, m_{e}, \varphi\right) \in I R^{4}\right\}$

\section{The Development Trend of Engine Idle Speed Control System}

Because the auto control system exist complex energy conversion process, the application of new technology introduced many new electric actuator,it has increased the complexity of the dynamic coupling [9], so it is difficult to through the quality of conservation, energy, and force (moment) the fundamental laws of physics and chemistry to establish precise mechanism model. Even to establish model, also due to the order time is too high, not suitable for the analysis and design of the control system.In addition, there are a lot of uncertain factors in the vehicle system, such as environmental temperature and pressure, surface friction coefficient, loading capacity, road slope, the differences between the prototype and mass products, spare parts aging and wear and tear, etc.In view of the difficult mechanism modeling, dynamics of strong coupling and uncertainty of complex systems,we can study the effective use of offline/online data system analysis and control method.So based on the data/mechanism modeling and control of the hybrid method is the future development direction of auto control,it has definite application value and scientific significance.Currently widely used engine average model is on the basis of a large number of experimental data of a model of experience [10], model accuracy is poorer, not fully reflect the working mechanism of engine and the parameters for different engine needs analysis, exploring the hybrid modeling method of variables between the deep understanding of mechanism of engine control system, developing new engine control method have important theoretical guiding significance.

\section{Summary}

The modeling method is commonly used in the simulation of engine at home and abroad, the engine which are used for auto control and performance analysis model.It includ static and dynamic modeling methods, such as engine nonlinear autoregressive model, the average model, the chart model and hybrid model.Engine as one of the main power supply, The valid of modeling directly affects the whole power system.Steady state modeling method based on the experimental data and 
complicated calculation is simple, but it is difficult to reflect the transient characteristics of the engine.Average model and dynamic model can well reflect the dynamic characteristics of engine, but model theory express complex, computing speed is relatively slow, modeling the required number of parameters is not easy to measure.At the same time, the theoretical model based on several assumptions, often difficult to ensure accuracy of model.In the actual process of modeling, we should make a trade-off between simplicity and accuracy of the model, select the appropriate modeling method for the specific problem.

\section{References}

[1] ThornhillM,Thomp Son S, Indano H A. Comparison of Idle Speed Control Schemes[J]. Control Engineering Practice,2000,(8): 1124 1130.

[2] Xing JianGuo, Xu Cangsu,Sun Youxian. Engine idle speed control NARX model and recognition [J]. Journal of internal combustion engine engineering, 2002, 23 (1) : $69 \sim 72$.

[3]G. De Nicolao, C. Rossi, R. Scattolini, M.Suffritti.Identification and idle speed control of internal combustion engines[J].Control Engineering Practice .1999:1061-1069.

[4] Wu Zhihu, Liu Zhiyuan, Pei Run. Modeling of the automobile engine idle speed and $\mathrm{H} \infty$ robust control with time-delay [J]. Journal of Harbin institute of technology, 2007:53-56.

[5] Woermann R J. Hardware in the Loop Simulator for Test and Development of Automotive Control Units[J]. SAE,961019.1996:15

[6]Yao Dongwei,Wu Feng. Used for engine control model of average discrete modeling method based on circular [J]. Journal of internal combustion engine, 2001-2001

[7] A.BALLUCHI, L.BENVENUTI,M.D.DI BENEDETTO. Idle speed control sythesis using an assume guarantee approach[J]. Nonline and Hybrid System in Automotive Control,2002:229 243

[8]A.BALLUCHI, L.BENVENUTI,M.D.DI BENEDETTO.Automotive engine control and hybrid system:challenge and opportunities[J].Proceeding of the IEEE.2000:888 912

[9] CHEN Hong, GONG Xun, HU Yun-Feng, LIU Qi-Fang, GAO Bing-Zhao, GUO Hong-Yan.Automotive Control: the State of the Art and Perspective [J]. ACTA AUTOMATICA SINICA.2013:322-342

[10]Wang Yaonan, Shen Yongpeng.Automotive gasoline engine electronic control system research present situation and the future [J]. Control theory and application. 2015:432-447 